\title{
Robot-assisted simple prostatectomy with temporary internal iliac arteries clamping: our preliminary results
}

\author{
Cristina Falavolti', Tommasangelo Petitti $^{2}$, Maurizio Buscarini ${ }^{2}$ \\ ${ }^{1}$ Department of Urology, Villa Betania Hospital, 00165 Rome, Italy. \\ ${ }^{2}$ Department of Hygiene, Campus Bio-Medico University of Rome, 00165 Rome, Italy.
}

Correspondence to: Dr. Cristina Falavolti, Department of Urology, Villa Betania Hospital, Via Pio IV 42, 00165 Rome, Italy. E-mail: c.falavolti@hotmail.com

How to cite this article: Falavolti C, Petitti T, Buscarini M. Robot-assisted simple prostatectomy with temporary internal iliac arteries clamping: our preliminary results. Mini-invasive Surg 2017;1:35-40.

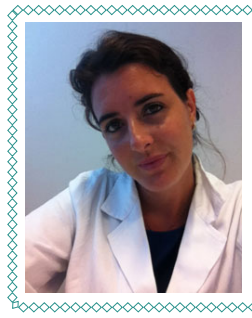

Dr. Cristina Falavolti is a young urologist working at Villa Betania Hospital of Rome. She received her medical degree from University Campus Bio-Medico of Rome in 2010 and completed her residency in Urology with honors at the same University in July 2016. She spent one year at Memorial Sloan Kettering - Cancer Center of New York City working with the bladder cancer group and training on robotic simulator. She published many original articles and participated as relator in many national and international meetings. She recently obtained the EBU certification. Her fields of interests are: (1) uro-oncological diseases (prostate, kidney and bladder cancers); (2) fusion biopsy for prostate cancer diagnosis; (3) minimally invasive surgery (laparoscopic and robotic) and treatment of benign diseases (BPH, stones).

\section{Article history:}

Received: $12-12-2016$

Accepted: 05-03-2017

Published: $31-03-2017$

Key words:

Benign prostatic hyperplasia,

clamping,

prostatectomy,

robotic

\section{ABSTRACT}

Aim: This study proposed the robot-assisted laparoscopic simple prostatectomy (RASP) as safe and reliable surgical option for the treatment of men with prostate size $>80 \mathrm{~mL}$. It was aimed to evaluate preoperative and postoperative results in RASP using a surgical variation to the standard technique: the temporary bilateral internal iliac arteries clamping. Methods: This study analyzed 18 patients underwent RASP with temporary clamping of bilateral internal iliac arteries. Procedures were performed by two surgeons in two different hospitals using the same surgical technique. Preoperative and postoperative data were collected and statistically analyzed. Results: The temporary clamping duration was less than 12 min during each adenoma's enucleation. Despite the vascular control, the median operating time was similar to RASP performed without iliac clamping. The results showed minimal blood loss, a median catheter duration of 5 days, a median duration of postoperative continuous catheter irrigation of $41 \mathrm{~h}$, and short hospitalization (3.2 days). A significant corellation was observed between the estimated blood loss and the duration of irrigation. Conclusion: RASP performed with bilateral vascular control, combined with the known benefits of minimally invasive surgery resulted in bleeding reduction. The minimal blood loss further reduces catheter duration, decreases continuous catheter irrigation and patient's hospitalization duration.

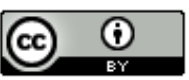

This is an open access article licensed under the terms of Creative Commons Attribution 4.0 International License (https://creativecommons.org/licenses/by/4.0/), which permits unrestricted use, distribution, and reproduction in any medium, as long as the original author is credited and the new creations are licensed under the identical terms.

For reprints contact: service@oaepublish.com

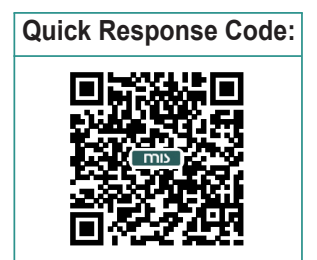




\section{INTRODUCTION}

Currently, minimally invasive surgery is the most common surgical approach for symptomatic benign prostatic hyperplasia (BPH). According to the EAU (European Association of Urology) guidelines, transurethral resection of the prostate represents the treatment of choice for men with prostate size < $80 \mathrm{~mL} \cdot{ }^{[1]}$ Some patients can be affected by complex conditions such as large adenoma (> $80 \mathrm{~mL}$ ) associated with moderate-to-severe lower urinary tract symptoms (LUTSs) and/or concomitant bladder diverticulum. In these cases, the endoscopic approach should be replaced by more invasive procedures. Open surgeries such as Trans-Vesical or Retropubic Adenomectomies are indicated in case of large adenoma and/or complex BPH but these techniques often show massive intraoperative blood loss and have the risk of blunt dissection particularly in the area around the apex and the urinary sphincter. ${ }^{[2-5]}$ For this reason, new techniques have been developed to combine the benefits of open simple prostatectomy with potential advantages of minimally invasive technique such as laparoscopic and robotic approaches. Robotassisted laparoscopic simple prostatectomy (RASP) represents a new treatment alternative, in expert hands, for these complex cases. This new alternative combines the advantages of laparoscopic surgery and three-dimensional vision, and increased digital degrees of freedom, resulting in surgical precision and improved results. ${ }^{[6,7]}$ The aim of the present study was to demonstrate the possibility of obtaining better intraoperative and postoperative results with RASP in terms of estimated blood loss, postoperative care and hospitalization using a surgical variation to the standard technique: the temporary bilateral internal iliac arteries clamping.

\section{METHODS}

\section{Patients and methods}

We retrospectively reviewed 18 cases of RASPs performed by two surgeons from March 2010 to May 2012 at two different hospitals. Each procedure was performed according to Sotelo's technique ${ }^{[7]}$ with the addition of the temporary clamping of internal iliac arteries. All patients were affected by severe symptomatic benign prostatic hyperplasia. Preoperative assessment included physical examination, International Prostate Symptom Score (IPSS) evaluation, serum creatinine, prostate specific antigen (PSA), uroflowmetry (except for the patients with an indwelling catheter) and volumetric suprapubic ultrasonography (US). The median preoperative IPSS was 25.2 (range 16-38). Fourteen patients (77.7\%) presented PSA value $<3.5 \mathrm{ng} / \mathrm{mL}$; four patients $(22.2 \%)$ presented higher PSA value and underwent previous trans-rectal ultrasound guided biopsy that confirmed the diagnosis of benign prostatic hyperplasia. Uroflowmetry revealed in all patients a peak flow < $10 \mathrm{~mL} / \mathrm{s}$ and an average flow $<5 \mathrm{~mL} / \mathrm{s}$. The median prostatic adenoma weight estimated preoperatively by US was $95 \mathrm{~g}$ (range 80-195). Based on these characteristics, our patients were classified as affected by complex prostatic hyperplasia and were scheduled to robot-assisted laparoscopic simple prostatectomy. We collected data about postoperative International IPSS evaluation, the duration of surgery, the estimated blood loss, postoperative care and hospitalization. Then, we statistically analyzed our results in a linear regression using the Fisher's test.

\section{Surgical technique}

All patients were placed in supine position and the table in deep Trendelenburg fashion. The surgeries were performed with a transperitoneal approach under general anesthesia in each case. Positioning included adequate padding of the pressure points on shoulder, back, legs and arms. The first trocar (camera port) was placed paraumbilical with the open (Hassan) technique. After the pneumoperitoneum was obtained, we performed a peritoneoscopy and placed the other robotic trocars under direct visualization. The abdomen was insufflated with a medium pressure of $12 \mathrm{mmHg}$ carbon dioxide gas. The ports were placed according to Sotelo et al. ${ }^{[7]}[$ Figure 1]: two robotic ports $(8 \mathrm{~mm})$ placed $9 \mathrm{~cm}$ from the camera port on an imaginary line joining the anterior superior iliac spine to the umbilicus; the third robotic port $(8 \mathrm{~mm})$ was placed in the left iliac fossa. Two additional ports were placed for the assistant instruments: one of $5 \mathrm{~mm}$ between the camera port and the first robotic arm on the upper right side and one of $12 \mathrm{~mm}$ in the right iliac fossa. We used both $0^{\circ}$ and $30^{\circ}$ optics, monopolar and bipolar robotic instruments. The 4-arm da Vinci $^{\circledR}$ Surgical System was docked and the intervention started with the development of the Retzius space and the isolation of the internal iliac arteries bilaterally using two vessel loops. Then, we cleared the anterior surface of the prostate capsule. In Figure 2 are showed the iliac arteries occluded with two Bulldog clamps. After clamping the arteries, a horizontal cystotomy, through the bladder mucosa, was made one centimeter cranial to the bladder neck. We dissected the adenoma along the subcapsular plane taking care of the prostatic capsule. We used two 2-0 vicryl stitches on the adenoma surface for traction. Extra care was taken at the apex of the prostate to avoid injury to the external sphincter. Accurate hemostasis was achieved before removing the prostatic adenoma en bloc in an Endo- 
Catch bag. The Bulldog clamps on the internal iliac arteries were removed; 2-0 monocryl running sutures were used for cystotomy closure in two layers. The prostatic fossa was then "trigonized" according to the technique described by Sotelo ${ }^{[7]}$ suturing the posterior edge of the bladder neck to the posterior edge of the urethra. An 18 French three-way Dufour catheter was placed and the balloon inflated to $30 \mathrm{~mL}$. Finally, we tested the bladder suture for leaks. We placed one drainage in the pelvis behind the bladder. The robotic arms were removed under vision and the abdominal wall was closed.

\section{RESULTS}

The demographics preoperative clinical data are showed in the Table 1. Patients' median age was 74 (range 65-88). The median postoperative IPSS at three months after surgery was 8 (range 3-13). The median operative duration was 205 min (range 120300 ) and the median estimated blood loss (EBL) was about $200 \mathrm{~mL}$ (range 100-350) irrespective of prostate weight. The median temporary clamping of internal iliac arteries duration average 12 min (range 11-14) during each adenoma's enucleation that were performed in about $10 \mathrm{~min}$. The median prostate weight on the pathological examination was $100 \mathrm{~g}$ (range 80195). Pathology revealed a benign glandular-stromal hyperplasia in all patients. The abdominal drain was removed on postoperative day 2. Continuous postoperative catheter irrigation was maintained for a median time of $41.5 \mathrm{~h}$ (range 18-55) in all patients. The median hospital stay was 3.2 days (range 2-6). The median catheter duration was 5.6 days (range 5-7). No patient required blood transfusion. Statistical analysis was performed between the estimated blood loss and the duration of continuous catheter irrigation. The logistic linear regression showed a significant statistical relation between these parameters $(P=$ 0.0395) [Figure 3]. Furthermore, patients did not present with symptoms of pelvic ischemia at the followup four months after surgery.

\section{DISCUSSION}

Although the definition of "large prostate" is still unclear, the surgical treatment of BPH is strictly dependent on prostate volume. For medium-size glands, transurethral resection of the prostate (TURP) is considered the gold standard. ${ }^{[1]}$ In fact, the EAU guidelines suggest TURP for men with prostate sizes $<80 \mathrm{~mL}$ and moderateto-severe lower urinary tract symptoms (LUTSs). ${ }^{[8]}$ Properly, a "large prostate" can be assumed as a gland $>80 \mathrm{~mL}$. In these cases, the surgical treatment is still controversial. Even though the lasers are becoming more popular especially for the treatment of mediumsmall prostate adenomas, open simple prostatectomy (OP) performed with either the Millin (retropubic) or the Freyer (open transvesical) technique is still an effective and reliable procedure ${ }^{[9]}$ for prostates $>80 \mathrm{~mL}$. Holmium laser enucleation of prostate (HoLEP) is seen as close rival of TURP. ${ }^{[10,11]}$ This procedure is showing good results in terms of blood loss, transfusion rates, and a hospital stay at the expense of longer operative time and postoperative dysuria. Despite its invasive nature, simple prostatectomies represent the $14-32 \%$ of all invasive procedures performed for $\mathrm{BPH}$ in Europe. ${ }^{[12]}$ Open procedure is often preferred in men who have a concomitant bladder condition, e.g. symptomatic bladder diverticulum, bladder calculus or inguinal hernia. Yet the disadvantages of open simple prostatectomy compared with transurethral resection are those of every open procedure such as the incision, the higher estimate blood loss and the necessity of

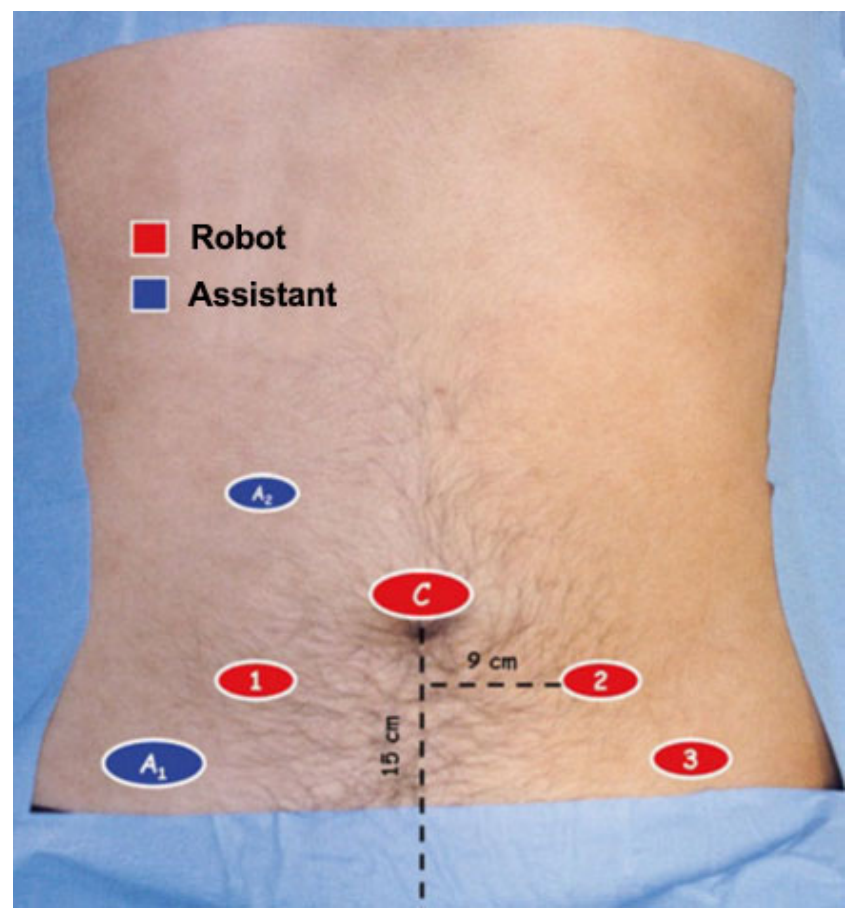

Figure 1: Port placement for simple prostatectomy. C: $12 \mathrm{~mm}$ robotic camera port; 1,2,3: $8 \mathrm{~mm}$ robotic working ports; $A_{1}: 12 \mathrm{~mm}$ assistant port; $\mathrm{A}_{2}: 5 \mathrm{~mm}$ assistant port

Table 1: Epidemiology and clinical data

\begin{tabular}{lcc}
\hline Characteristics & Median & Range \\
\hline Age (years) & 74.3 & $65-88$ \\
Operative duration (min) & 205 & $120-300$ \\
Ematic blood loss (mL) & 200 & $100-350$ \\
Catheterization (days) & 5.6 & $5-7$ \\
Drainage (days) & 2 & 2 \\
Hospitalization (days) & 3.2 & $2-6$ \\
Prostate weight (g) & 100 & $80-195$ \\
Preoperative IPSS & 25.2 & $16-38$ \\
Postoperative IPSS & 8 & $3-13$ \\
Continuous catheter irrigation (h) & 41.5 & $18-55$ \\
\hline
\end{tabular}

IPSS: International Prostatic Symptoms Score 


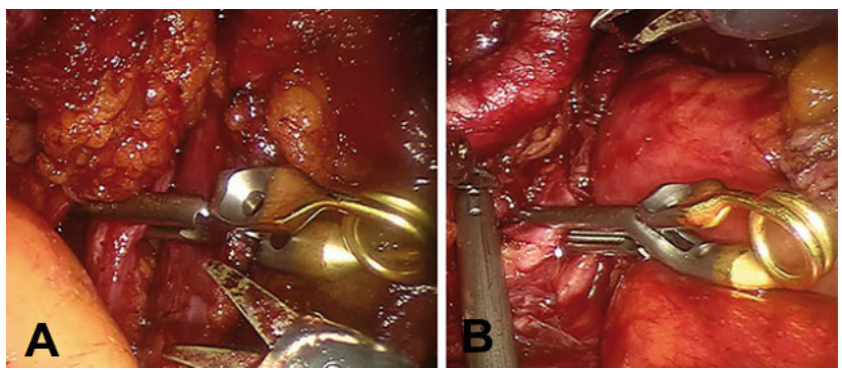

Figure 2: Occlusion of bilateral internal iliac arteries. A: on the right side; $\mathrm{B}$ : on the left side

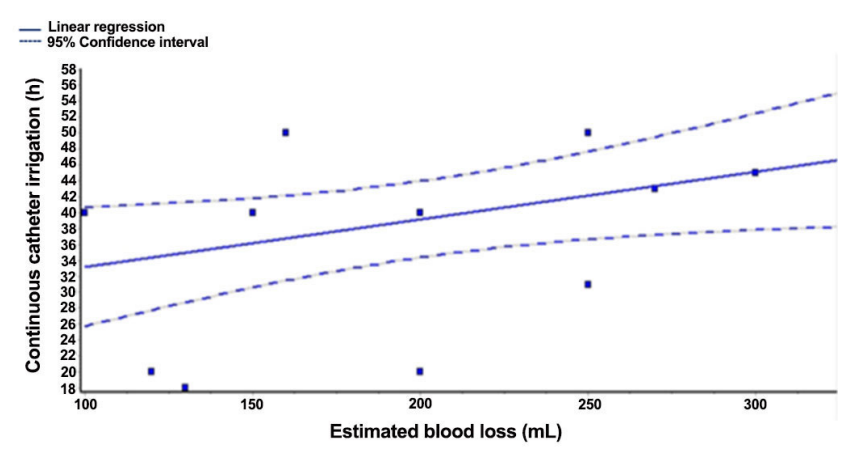

Figure 3: Logistic linear regression ( $X$ intercept: estimated blood loss; $Y$ intercept: hours of continuous catheter irrigation)

transfusions, the prolonged hospital stay with a longer convalescence period. Transfusion rate of $0 \%$ to $57 \%$ has been reported due to excessive bleeding. ${ }^{[9]}$ In 20th century, minimally invasive surgeries have been developed to limit the blood loss, to provide a shorter hospitalization and urethral catheterization, and to allow minimal postoperative pain and complications. So, the minimally invasive approach for BPH is replacing open surgery. Both laparoscopic and robotic techniques have those benefits. The first laparoscopic simple prostatectomy (LSP) was first described by Mariano et al. ${ }^{[13]}$ in 2002. This procedure combined the benefits of open simple prostatectomy (OSP) with the potential advantages of a minimally invasive approach. Subsequently, several papers in the literature started to compare the open surgery and laparoscopic approach. Porpiglia and colleagues showed that the only benefit of laparoscopic simple prostatectomy was a less intraoperative blood loss. The other parameters such as: operation duration, postoperative pain, catheterization duration and hospitalization were almost the same between the laparoscopy group and the open procedures. ${ }^{[14]}$ Also McCullough and associates compared the same two groups. In their study, the operation duration was significantly longer in laparoscopy group but catheterization and hospitalization were significantly shorter. There was no difference between bleeding and irrigation periods. ${ }^{[15]}$ Case series in the literature are few but the reported results showed that laparoscopic adenomectomy is a reasonable alternative to the open prostatectomy. ${ }^{[16]}$
With continued expansions in the field of robotic surgery, urologists are now available to combine the advantages of laparoscopic procedures such as shorter hospitalization, less total amount of blood loss, more efficient intracorporeal suturing and cosmetic results and those of robotic surgery: 6 degrees of freedom, dexterity enhancement, $3-D$ vision, and tremor filtering. The feasibility of robotic-assisted adenomectomy was confirmed by Sotelo et al. ${ }^{[7]}$ in 2008 . Their data showed that the patients who underwent robotic-assisted prostatectomy had significant improvements in urinary flow, postvoid residual measurements, IPSS scores and cosmetic results than those who had undergone open surgery. The operative times, the hospitalization, the low dose of analgesics required and the minimal blood loss calculated, were similar to those seen in laparoscopic series. ${ }^{[7,15,17-19]}$ Matei et al. ${ }^{[20]}$ have recently reported the series of 35 patients underwent RASP. Although Matei and colleagues presented the largest series of RASP, we reported our series of 18 patients treated with RASP and early vascular control: the temporarily bilateral internal iliac arteries clamping. The early vascular control makes the procedure a safer alternative for treating $\mathrm{BPH} .{ }^{[21]}$ Our results showed a median estimated blood loss (EBL) of $200 \mathrm{~mL}$ that is less than the median value of the other series (> $300 \mathrm{~mL}){ }^{[20]}$ No transfusions have been necessary. Despite the vascular control, our median operating time is superimposable to the RASP performed without clamping the iliac arteries. Furthermore, we reported a median catheter duration of 5.6 days (range 5-7 days) that is lower than Matei et al. ${ }^{[20]}$ Our results showed also a significant statistical relation between the EBL and the duration of continuous catheter irrigation $(P=$ 0.0395 ) with median hospitalization of 3.2 days (range 2-6 days). The early vascular control reduces the intraoperative blood loss and possibly the necessity of transfusion. Consequently, also the catheter duration and the hospitalization can be shorter and costs decrease. Our results agree with those of more recent larger series. ${ }^{[22-23]}$ The most important possible side effect of clamping the internal iliac arteries is the pelvic ischemia. It can manifest in different ways and often the symptoms are transient and resolve with time. It is very important to take care not to prolong the arteries clamping for a long time. In these cases, patients can present serious complications such as colorectal ischemia, gluteal necrosis and neurological deficit or buttock claudication and sexual dysfunction. ${ }^{[21]}$ The intensity of possibly complication depends on the status of collateral circulation around the internal iliac artery and/or the presence of stenosis of the origin of the remaining internal iliac arter ${ }^{[24]}$ In our series, the internal iliac arteries clamping was performed for less than 12 min in each surgery and we did not have 
cases of pelvic ischemia manifestation. Nevertheless, robotic-assisted laparoscopic simple prostatectomy is considered a new surgical approach and prospective future comparative studies are needed to determine the efficacy of this procedure. Our paper showed the preliminary results of robotic assisted laparoscopic simple prostatectomy with temporary internal iliac arteries clamping and we believe that this approach could be a safe surgical option for the treatment of large prostatic adenomas $(>80 \mathrm{~mL}$ ) reducing the estimated blood loss and decreasing needed postoperative care.

In conclusion, the surgical treatment of large prostatic adenomas remains a controversial issue. The minimally invasive approach for $\mathrm{BPH}$ is replacing open surgery. Both laparoscopic and robotic techniques have benefits. We propose robotic assisted laparoscopic simple prostatectomy with early vascular control as technique able to associate the benefits of minimally invasive surgery with those of minimal estimated blood loss. We believe that this approach could be a safe surgical option for the treatment of large prostatic adenomas (> $80 \mathrm{~mL}$ ) especially for surgeons at the beginning of their learning curve.

\section{Authors' contributions}

Original idea and data collection: C. Falavolti

Data and statistical analysis: T. Petitti

Drafting of the text: M. Buscarini

\section{Financial support and sponsorship None.}

\section{Conflicts of interest}

There are no conflicts of interest.

\section{Patient consent}

The informed consent was obtained from all patients.

\section{Ethics approval}

This article is waived for ethical approval in authors' affiliations.

\section{REFERENCES}

1. Gratzke C, Bachmann A, Descazeaud A, Drake MJ, Madersbacher S, Mamoulakis C, Oelke M, Tikkinen KA, Gravas S. EAU guidelines on management on benign prostatic hyperplasia. Eur Urol 2015;67:1099-109.

2. John H, Bucher C, Engel N, Fischer B, Fehr JL. Preperitoneal robotic prostate adenomectomy. Urology 2009;73:811-5.

3. Gratzke C, Schlenker B, Seitz M, Karl A, Hermanek P, Lack N, Stief CG, Reich O. Complications and early postoperative outcome after open prostatectomy in patients with benign prostatic enlargement: results of a prospective multicenter study. J Urol 2007;177:1419-22.

4. Ou R, You M, Tang P, Chen H, Deng X, Xie K. A randomized trial of transvesical prostatectomy versus transurethral resection of the prostate for prostate greater than $80 \mathrm{~mL}$. Urology 2010;76:958-61.

5. Alivizatos G, Skolarikos A, Chalikopoulos D, Papachristou C, Sopilidis O, Dellis A, Kastriotis I, Deliveliotis C. Transurethral photoselective vaporization versus transvesical open enucleation for prostatic adenomas $>80 \mathrm{~mL}$ : 12 -mo results of a randomized prospective study. Eur Urol 2008;54:427-37.

6. Sergi F, Falavolti C, Bove AM, Buscarini M. Robotic-assisted laparoscopic simple prostatectomy and bladder diverticulectomy with temporary clamping of internal iliac arteries. $J$ Robot Surg 2014;8:81-3.

7. Sotelo R, Clavijo R, Carmona O, Garcia A, Banda E, Miranda M, Fagin R. Robotic simple prostatectomy. J Urol 2008;179:513-5.

8. Madersbacher S, Alivizatos G, Nordling J, Sanz CR, Emberton M, de la Rosette JJ. EAU 2004 guidelines on assessment, therapy and followup of men with lower urinary tract symptoms suggestive of benign prostatic obstruction (BPH guidelines). Eur Urol 2004;46:547-54.

9. Tubaro A, Carter S, Hind A, Vicentini C, Miano L. A prospective study of the safety and efficacy of suprapubic transvesical prostatectomy in patients with benign prostatic hyperplasia. J Urol 2001;166:172-6.

10. Jhanwar A, Sinha RJ, Bansal A, Prakash G, Singh K, Singh V. Outcomes of transurethral resection and holmium laser enucleation in more than $60 \mathrm{~g}$ of prostate: a prospective randomized study. Urol Ann 2017;9:45-50.

11. Naspro R, Gomez Sancha F, Manica M, Meneghini A, Ahyai S, Aho T, Fiori C, Vavassori I, DA Pozzo LF, Pansadoro V, Montorsi F, Herrmann TR. From "gold standard" resection to reproducible "future standard" endoscopic enucleation of the prostate (EEP): what we know about anatomical enucleation. Minerva Urol Nefrol 2017; doi: 10.23736/S0393-2249.17.02834-X.

12. Reich O, Gratzke C, Stief CG. Techniques and long-term results of surgical procedures for BPH. Eur Urol 2006;49:970-8.

13. Mariano MB, Graziottin TM, Tefilli MV. Laparoscopic prostatectomy with vascular control for benign prostatic hyperplasia. J Urol 2002;167:2528-9.

14. Porpiglia F, Terrone C, Renard J, Grande S, Musso F, Cossu M, Vacca F, Scarpa RM. Transcapsular adenomectomy (Millin): a comparative study, extraperitoneal laparoscopy versus open surgery. Eur Urol 2006;49:120-6.

15. McCullough TC, Heldwein FL, Soon SJ, Galiano M, Barret E, Cathelineau X, Prapotnich D, Vallancien G, Rozet F. Laparoscopic versus open simple prostatectomy: an evaluation of morbidity. $J$ Endourol 2009;23:129-33

16. Oktay B, Koc G, Vuruskan H, Danisoglu ME, Kordan Y. Laparoscopic extraperitoneal simple prostatectomy for benign prostate hyperplasia: a two-year experience. Urol J 2011;8:107-12.

17. Yuh B, Laungani R, Perlmutter A, Eun D, Peabody JO, Mohle JL, Stricker H, Guru KA. Robot-assisted Millin's retropubic prostatectomy: case series. Can J Urol 2008;15:4101-5.

18. John H, Bucher C, Engel N, Fischer B, Fehr JL. Preperitoneal robotic prostate adenomectomy. Urology 2009;73:811-5.

19. Vora A, Mittal S, Hwang J, Bandi G. Robot-assisted simple prostatectomy: multi-institutional outcomes for glands larger than 100 grams. J Endourol 2012;26:499-502.

20. Matei DV, Brescia A, Mazzoleni F, Spinelli M, Musi G, Melegari S, Galasso G, Detti S, de Cobelli O. Robot-assisted simple prostatectomy (RASP): does it make sense? BJU Int 2012;110:E972-9.

21. Shaheen A, Quinlan D. Feasibility of open simple prostatectomy with early vascular control. BJU Int 2004;93:349-52.

22. Pokorny M, Novara G, Geurts N, Dovey Z, De Groote R, Ploumidis A, Schatteman P, de Naeyer G, Mottrie A. Robot-assisted simple prostatectomy for treatment of lower urinary tract symptoms secondary to benign prostatic enlargement: surgical technique and outcomes in a high-volume robotic centre. Eur Urol 2015;68:451-7. 
23. Autorino R, Zargar H, Mariano MB, Sanchez-Salas R, Sotelo RJ, Chlosta PL, Castillo O, Matei DV, Celia A, Koc G, Vora A, Aron M, Parsons JK, Pini G, Jensen JC, Sutherland D, Cathelineau X, Nuñez Bragayrac LA, Varkarakis IM, Amparore D, Ferro M, Gallo G, Volpe A1, Vuruskan H, Bandi G, Hwang J, Nething J, Muruve N, Chopra S, Patel ND, Derweesh I, Champ Weeks D, Spier R, Kowalczyk K, Lynch J, Harbin A, Verghese M, Samavedi S, Molina WR, Dias E, Ahallal Y, Laydner H, Cherullo E, De Cobelli O, Thiel DD, Lagerkvist
M, Haber GP, Kaouk J, Kim FJ, Lima E, Patel V, White W, Mottrie A, Porpiglia F. Perioperative outcomes of robotic and laparoscopic simple prostatectomy: a European-American multi-institutional analysis. Eur Urol 2015;68:86-94.

24. Yano OJ, Morrissey N, Eisen L, Faries PL, Soundararajan K, Wan S, Teodorescu V, Kerstein M, Hollier LH, Marin ML. Intentional internal iliac artery occlusion to facilitate endovascular repair of aortoiliac aneurysms. J Vasc Surg 2001;34:204-11. 